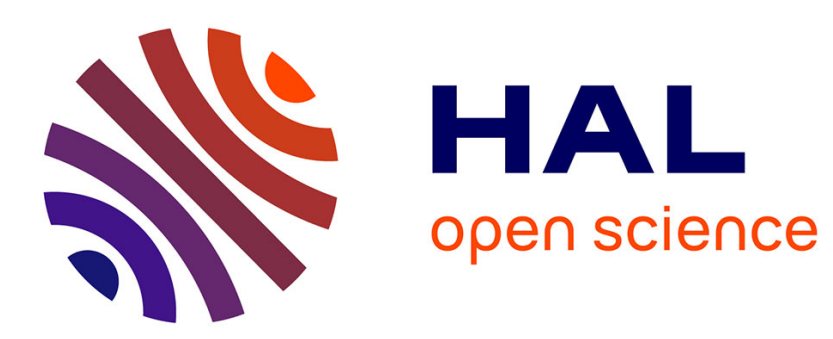

\title{
Asymptotics of Subexponential Max Plus Networks : the Stochastic Event Graph Case
}

François Baccelli, Serguei Foss, Marc Lelarge

\section{To cite this version:}

François Baccelli, Serguei Foss, Marc Lelarge. Asymptotics of Subexponential Max Plus Networks: the Stochastic Event Graph Case. [Research Report] RR-4952, INRIA. 2003. inria-00071627

\section{HAL Id: inria-00071627 \\ https://hal.inria.fr/inria-00071627}

Submitted on 23 May 2006

HAL is a multi-disciplinary open access archive for the deposit and dissemination of scientific research documents, whether they are published or not. The documents may come from teaching and research institutions in France or abroad, or from public or private research centers.
L'archive ouverte pluridisciplinaire HAL, est destinée au dépôt et à la diffusion de documents scientifiques de niveau recherche, publiés ou non, émanant des établissements d'enseignement et de recherche français ou étrangers, des laboratoires publics ou privés. 
INSTITUT NATIONAL DE RECHERCHE EN INFORMATIQUE ET EN AUTOMATIQUE

\title{
Asymptotics of Subexponential Max Plus Networks the Stochastic Event Graph Case
}

\author{
François Baccelli — Serguei Foss — Marc Lelarge
}

\section{$\mathbf{N}^{\circ} 4952$}

Octobre 2003

THÈME 1 



\title{
RIN RIA
}

\section{Asymptotics of Subexponential Max Plus Networks the Stochastic Event Graph Case}

\author{
François Baccelli $^{*}$, Serguei Foss ${ }^{\dagger}$, Marc Lelarge ${ }^{\ddagger}$ \\ Thème 1 -Réseaux et systèmes \\ Projet TREC \\ Rapport de recherche $n^{\circ} 4952$ - Octobre 2003 -25 pages
}

\begin{abstract}
We calculate the exact tail asymptotics of stationary response times for open stochastic event graphs, in the irreducible and reducible cases. These networks admit a representation as (max,plus)-linear systems in a random medium. We study the case of renewal input and i.i.d service times with subexponential distributions. We show that the stationary response times have tail asymptotics of the same order as the integrated tail of service times. The mutiplicative constants only involve the intensity of the arrival process and the (max,plus)-Lyapunov exponents of certain sequences of (max,plus)-matrices associated to the event graph.
\end{abstract}

Key-words: Open queueing network, stochastic event graph, sub-exponential random variable, heavy tail, integrated tail, Veraverbeke's theorem, (max,plus)-network, tandem queue.

\footnotetext{
* INRIA-ENS, ENS, 45 rue d'Ulm, 75005 Paris, France, Francois.Baccelli@ens.fr

$\dagger$ Institute of Mathematics, 630090 Novosibirsk, Russia \& Department of Actuarial Mathematics and Statistics, HeriotWatt University, Edinburgh, UK, S.Foss@ma.hw.ac.uk, the work of this author was supported by INTAS grant no 265.

$\ddagger$ INRIA-ENS, ENS, 45 rue d’Ulm, 75005 Paris, France, Marc.Lelarge@ens.fr
} 


\section{Asymptotiques de réseaux (max,plus) sous-exponentiels Le cas des graphes d'événements}

Résumé : Nous calculons le comportement asymptotique de la queue de la distribution stationnaire du temps de réponse d'un graphe d'événements stochastique ouvert, tant dans le cas réductible que dans les cas irréductible. Ces réseaux admettent une représentation sous la forme d'un système (max,plus)-linéaire en milieu aléatoire. Nous étudions le cas où le processus d'entrée est un processus de renouvellement et où les temps de services sont i.i.d. et de distribution sous-exponentielle. Nous montrons que les temps de réponse stationnaires ont une queue de distribution du même ordre que l'intégrale de la queue des distributions des temps de service. Les constantes multiplicatives font simplement intervenir l'intensité du processus d'entrée et les exposants de Lyapounov (max,plus) de certaines suites de matrices (max,plus) associées au graphe d'événements.

Mots-clés : Réseau ouvert, graphe d'événéments stochastique, variable aléatoire sous-exponentielle, distribution à queue lourde, distribution intégrée, théorème de Veraverbeke, réseau (max,plus), files d'attente en série. 


\section{Introduction}

This paper is focused on the derivation of the tail asymptotics of the steady state end-to-end response times in open, single input, stochastic event graphs [3], a class of networks which are known to admit a (max,plus)-linear representation.

To the best of our knowledge, within this class of networks, under subexponential statistical assumptions, exact asymptotics are only known for the following special cases:

- the case of dimension 1; this type of asymptotics is known as Pake's [10] or Veraverbeke's theorem [11], and most often expressed as a property of the waiting or response times in the $\mathrm{G} / \mathrm{G} / 1$ queue (this can also be seen as a property of extrema of random walks);

- the case of irreducible event graphs [7], a first class of networks with general dimension that contains the $\mathrm{G} / \mathrm{G} / 1$ queue as a special case;

- the case of tandem queues [5], a class of reducible event graphs with a specific linear topology, which also contains the G/G/1 queue as a special case.

The exact asymptotic for the maximal dater of a generalized Jackson network is the subject of a paper in preparation [6]. This list only concerns open networks. There are also some recent results on asymptotics of closed subexponential event graphs in [2].

The aim of the present paper is to extend the results on open networks by giving an asymptotic theorem for event graphs with general, possibly reducible topology and with general dimension. For this, we use a theorem, called the typical event theorem, established in [5], which shows that for all subexponential monotone separable networks, a large deviation from the mean behavior is triggered by a single large service time, in some station of the network, at some distant epoch in the past.

The paper is structured as follows: Section 2 summarizes the different frameworks that are needed to state the problem and the results. We first recall the definition of monotone separable networks introduced in [4]. We then present the class of (max,plus)-linear networks and the subclass of event graphs. We show that event graphs belong to the monotone separable networks class. This first section is completely algebraic and does not require any stochastic assumption.

The stochastic assumptions are introduced in Section 3, whereas Section 4 gives the main result of this paper, Theorem 1, which expresses the tail asymptotics of the steady state end-to-end response time in function of the integrated tail of the service (or firing) times of the network, the arrival intensity and the (max,plus)-Lyapunov exponents of the communication classes of the network. 


\section{General Framework}

\subsection{Monotone Separable Networks: $\mathcal{M S}$-Net.}

In the present paper, by network we will understand the following:

- An input point process $N$, with an infinite number of points $\left\{T_{n}\right\}$; for all $m \leq n$, we will benote by $N_{[m, n]}$ the $[m, n]$ restriction of $N$, namely the point process with points $\left\{T_{l}\right\}_{m \leq l \leq n}$.

- A mapping $X($.$) which associates, to each (finite or infinite) point process, the time of last$ activity in the network when fed by this point process. We assume the following on this mapping:

- There exists a sequence $\left\{\xi_{n}\right\}$, with $\xi_{n}$ in some measurable space $\mathcal{M}$, describing service times and routing decisions, and a set of functions $\left\{f_{l}\right\}, f_{l}: \mathbb{R}^{l} \times \mathcal{M}^{l} \rightarrow \mathbb{R}$, such that

$$
X\left(N_{[m, n]}\right)=f_{n-m+1}\left\{\left(T_{l}, \xi_{l}\right), m \leq l \leq n\right\} .
$$

- For all finite $m \leq n, X\left(N_{[m, n]}\right)$ is finite.

We say that such a network is monotone-separable if for all $N$ :

1. Causality: for all $m \leq n$,

$$
X\left(N_{[m, n]}\right) \geq T_{n}
$$

2. External monotonicity: for all $m \leq n$,

$$
X\left(N_{[m, n]}^{\prime}\right) \geq X\left(N_{[m, n]}\right),
$$

whenever $N^{\prime} \equiv\left\{T_{n}^{\prime}\right\}$ is such that $T_{n}^{\prime} \geq T_{n}$ for all $n$, a property which we will write $N^{\prime} \geq N$ for short;

3. Homogeneity: for all $c \in \mathbb{R}$ and for all $m \leq n$

$$
X\left(N_{[m, n]}+c\right)=X\left(N_{[m, n]}\right)+c ;
$$

4. Separability: if for all $m \leq l<n, X\left(N_{[m, l]}\right) \leq T_{l+1}$, then

$$
X\left(N_{[m, n]}\right)=X\left(N_{[l+1, n]}\right) .
$$

By definition, the $[m, n]$ maximal dater is $Z_{[m, n]}(N)=X\left(N_{[m, n]}\right)-T_{n}=X\left(N_{[m, n]}-T_{n}\right)$. 


\section{2 (max,plus)-Linear Systems}

Definition 1 The (max,plus) semi-ring $\mathbb{R}_{\max }$ is the set $\mathbb{R} \cup\{-\infty\}$, equipped with max, written additively (i.e. $a \oplus b=\max (a, b)$ ) and the usual sum, written multiplicatively (i.e. $a \otimes b=a+b$ ). The zero element is denoted $\epsilon=-\infty$.

For matrices of appropriate sizes, we define $(A \oplus B)^{(i, j)}=A^{(i, j)} \oplus B^{(i, j)}=\max \left(A^{(i, j)}, B^{(i, j)}\right)$, $(A \otimes B)^{(i, j)}=\bigoplus_{k} A^{(i, k)} \otimes B^{(k, j)}=\max _{k}\left(A^{(i, k)}+B^{(k, j)}\right)$.

Let $s$ be an arbitrary fixed natural number. Assume the following to be given:

- $\left\{T_{n}, n \in \mathbb{N}\right\}$, where $T_{n} \in \mathbb{R}$, the arrival time sequence;

- $\left\{A_{n}, n \in \mathbb{N}\right\}$, where $A_{n}$ is a $s \times s$ matrix;

- $\left\{B_{n}, n \in \mathbb{N}\right\}$, where $B_{n}$, is a $s$-dimensional vector.

The associated (max,plus)-linear recurrence is that with state variable sequence $\left\{X_{n}, n \in \mathbb{N}\right\}$, where $X_{n}$ is a $s$-dimensional vector, which satisfies the evolution equation:

$$
X_{n+1}=A_{n+1} \otimes X_{n} \oplus B_{n+1} \otimes T_{n+1} .
$$

We assume w.l.o.g. that $A_{n}$ has no null column $\left(=(\epsilon \ldots \epsilon)^{\prime}\right)$ and that if the $i$-th line of $A_{n}$ is null, then $B_{n}^{(i)} \geq 0$.

To each (max,plus)-linear recurrence, one associates a network in the sense of the last section, with $\xi_{n}=\left(A_{n}, B_{n}\right)$ and

$$
X\left(N_{[m, n]}\right)=\bigoplus_{1 \leq i \leq s} \bigoplus_{m \leq k \leq n}\left(D_{[k, n]} \otimes B_{k} \otimes T_{k}\right)^{(i)},
$$

where for $k<n, D_{[k, n]}=\bigotimes_{j=n}^{k+1} A_{j}=A_{n} \otimes \cdots \otimes A_{k+1}$ and $D_{[n, n]}=E$, the identity matrix (the matrix with all its diagonal entries equal to 0 and all its non-diagonal ones equal to $\epsilon$ ). If one defines

$$
Y_{[m, n]}=\bigoplus_{m \leq k \leq n} D_{[k, n]} \otimes B_{k} \otimes T_{k},
$$

it is easy to check that $Y_{[m, m]}=B_{m} \otimes T_{m}$, that for all $n \geq m$,

$$
Y_{[m, n+1]}=A_{n+1} \otimes Y_{[m, n]} \oplus B_{n+1} \otimes T_{n+1}
$$

and that $X\left(N_{[m, n]}\right)=\max _{i}\left(Y_{[m, n]}\right)^{(i)}$.

For all real numbers $x$, let $\mathbf{x}$ denote the vector with all its entries equal to $x$.

Lemma 1 The network associated with a (max,plus)-linear recurrence is monotone-separable provided $A_{n} \otimes \mathbf{0} \leq B_{n}$ for all $n$. 


\section{Proof:}

The first three properties are immediate. Let us prove that separability holds under the last assumption. If $X\left(N_{[m, l]}\right) \leq T_{l+1}$, then $Y_{[m, l]} \leq \mathbf{T}_{\mathbf{l + 1}}$. So by monotonicity, $A_{l+1} \otimes Y_{[m, l]} \leq A_{l+1} \otimes \mathbf{T}_{\mathbf{l}+\mathbf{1}}$. This and the assumption $A_{l+1} \otimes \mathbf{0} \leq B_{l+1}$ imply $Y_{[m, l+1]}=B_{l+1} \otimes T_{l+1}=Y_{[l+1, l+1]}$. An immediate induction then shows that more generally, $Y_{[m, n]}=Y_{[l+1, n]}$, for all $n \geq l+1$. This in turn implies separability.

\subsection{Event Graphs: $\mathcal{E} \mathcal{G}$}

Consider a bipartite oriented graph $\mathcal{G}$ with two types of nodes: transitions (denoted by bars) and places (denoted by circles), and with an integer marking of each place. We will only consider the class of event graphs, which is the class of such bipartite graphs where each place has exactly one upstream and one downstream transition. An example of such a graph is provided below where the integer marking of a place (here 0 or 1 ) is depicted by tokens. We will also assume that the event graph is live, namely that there is no circuit with only places of zero marking.

A transition without predecessor is called a source; similarly a transition with no successor is called a sink; we will consider networks that have exactly one source and one sink and we will adopt the following notation:

- For the source:

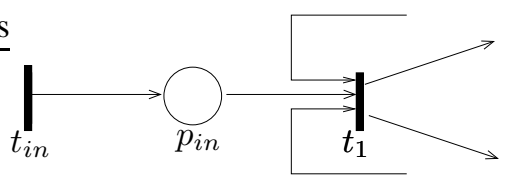

- For the sink:

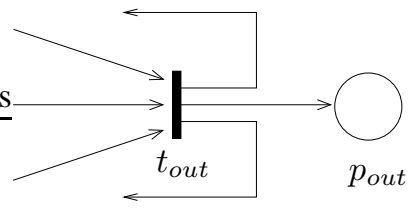

Consider an event graph, together with ( $\mathcal{T}$ denote the set of transitions):

- a sequence of non-negative, real variables $\sigma_{n}^{i}, i \in \mathcal{T}, n \geq 0$;

- an increasing sequence of real variables $T_{n}, n \geq 0$.

We show below that to such a triple, one can associate a (max,plus)-linear recurrence of type (2). 
For this, take $K=|\mathcal{T}|$, and adopt a numbering of coordinates such that coordinate 1 is the source and $K$ the sink. For all $m=0, \ldots, L$, where $L$ is the maximal value of the initial marking, define $a_{m}(n)$ to be the $K \times K$ matrix with entries

$$
\left(a_{m}(n)\right)^{(i, j)}= \begin{cases}\sigma_{n}^{i} & \text { if there is two hop path from } j \text { to } i \text { with a place with marking } m \\ \varepsilon & \text { otherwise. }\end{cases}
$$

Let $b$ the $K$-dimensional vector with all its entries equal to $\varepsilon$, but the first, which is equal to 0 . Let then $x_{n}$ be the sequence of $K$-dimensional vectors defined by the recurrence relation

$$
x_{n}=a_{0}(n) \otimes x_{n} \oplus \cdots \oplus a_{L}(n) \otimes x_{n-L} \oplus b \otimes T_{n} .
$$

The reduction to a (max,plus)-recurrence is then obtained as follows: the matrix $a_{0}$ can be assumed to be strictly triangular w.l.o.g. thanks to the liveness assumption (see [3]). Therefore the matrix

$$
a_{0}(n)^{*}=E \oplus a_{0}(n) \oplus a_{0}(n)^{2} \oplus \ldots
$$

is well defined and when defining $\bar{a}_{i}(n)=a_{0}(n)^{*} \otimes a_{i}(n)$ and $\bar{b}(n)=a_{0}(n)^{*} \otimes b$, we obtain

$$
x_{n}=\bar{a}_{1}(n) \otimes x_{n-1} \oplus \cdots \oplus \bar{a}_{L}(n) \otimes x_{n-L} \oplus \bar{b}(n) \otimes T_{n} .
$$

Then, with the following notation

$$
X_{n}=\left(\begin{array}{c}
x_{n-L+1} \\
\vdots \\
x_{n}
\end{array}\right) \text {, }
$$

we get the desired equation, namely $X_{n}=A_{n} X_{n-1} \oplus B_{n} T_{n}$, when taking

$$
A_{n}=\left(\begin{array}{ccccc}
\epsilon & 0 & & & \\
\vdots & & \ddots & & \\
\vdots & & & \ddots & \\
\epsilon & & & & 0 \\
\bar{a}_{L}(n) & \bar{a}_{L-1}(n) & \ldots & \ldots & \bar{a}_{1}(n)
\end{array}\right), \quad B_{n}=\left(\begin{array}{c}
\epsilon \\
\vdots \\
\vdots \\
\epsilon \\
\bar{b}(n)
\end{array}\right) .
$$

So to each event graph, one can associate a (max,plus)-linear recurrence and therefore a network.

Remark 1 One can drop coordinate $i$ if column $i$ has only $\epsilon$ entries (indeed, in this case coordinate $i$ is never used in the recursion). We can drop coordinates successively. We will not do this for the last column, which is associated to the last activity. 
Here is an example. Consider the following graph:

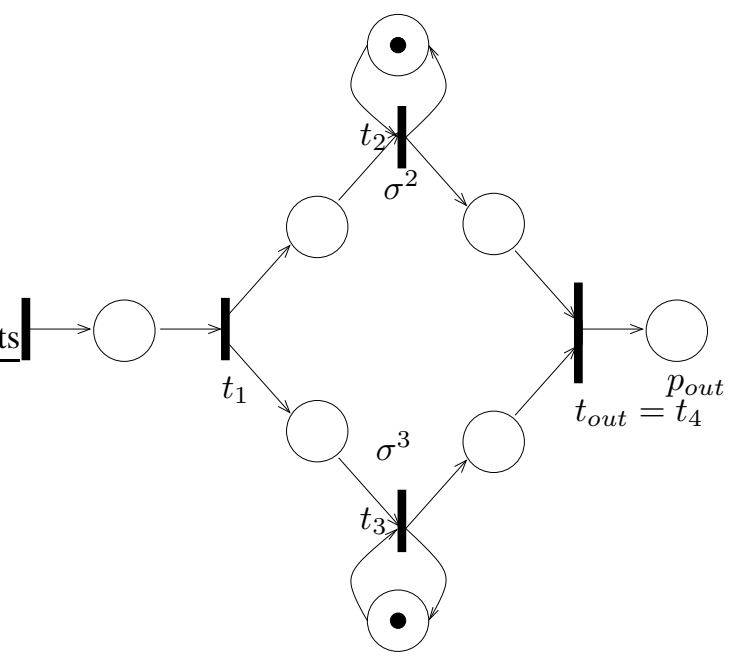

and take all sigma's equal to 0 but for transitions 2 and 3 for which we take some sequences $\sigma_{n}^{2}$ and $\sigma_{n}^{3}$ respectively. Here, $K=4, L=1$ and the matrices are

$$
a_{0}(n)=\left(\begin{array}{cccc}
\epsilon & \epsilon & \epsilon & \epsilon \\
\sigma_{n}^{2} & \epsilon & \epsilon & \epsilon \\
\sigma_{n}^{3} & \epsilon & \epsilon & \epsilon \\
\epsilon & 0 & 0 & \epsilon
\end{array}\right), \quad a_{1}(n)=\left(\begin{array}{cccc}
\epsilon & \epsilon & \epsilon & \epsilon \\
\epsilon & \sigma_{n}^{2} & \epsilon & \epsilon \\
\epsilon & \epsilon & \sigma_{n}^{3} & \epsilon \\
\epsilon & \epsilon & \epsilon & \epsilon
\end{array}\right)
$$

The evolution equations are:

$$
\begin{aligned}
x_{n}^{(1)} & =T_{n}, \\
x_{n}^{(2)} & =\left[x_{n}^{(1)} \oplus x_{n-1}^{(2)}\right] \otimes \sigma_{n}^{2}, \\
x_{n}^{(3)} & =\left[x_{n}^{(1)} \oplus x_{n-1}^{(3)}\right] \otimes \sigma_{n}^{3}, \\
x_{n}^{(4)} & =x_{n}^{(2)} \oplus x_{n}^{(3)} .
\end{aligned}
$$

Denoting $\sigma_{n}^{i \vee j}=\max \left(\sigma_{n}^{i} ; \sigma_{n}^{j}\right)$, we get:

$$
\begin{aligned}
x_{n}^{(1)} & =T_{n}, \\
x_{n}^{(2)} & =x_{n-1}^{(2)} \otimes \sigma_{n}^{2} \oplus T_{n} \otimes \sigma_{n}^{2}, \\
x_{n}^{(3)} & =x_{n-1}^{(3)} \otimes \sigma_{n}^{3} \oplus T_{n} \otimes \sigma_{n}^{3}, \\
x_{n}^{(4)} & =x_{n-1}^{(2)} \otimes \sigma_{n}^{2} \oplus x_{n-1}^{(3)} \otimes \sigma_{n}^{3} \oplus T_{n} \otimes\left[\sigma_{n}^{2 \vee 3}\right] .
\end{aligned}
$$


So we have a (max,plus)-linear recurrence with

$$
A_{n}=\left(\begin{array}{cccc}
\epsilon & \epsilon & \epsilon & \epsilon \\
\epsilon & \sigma_{n}^{2} & \epsilon & \epsilon \\
\epsilon & \epsilon & \sigma_{n}^{3} & \epsilon \\
\epsilon & \sigma_{n}^{2} & \sigma_{n}^{3} & \epsilon
\end{array}\right) \quad B_{n}=\left(\begin{array}{c}
0 \\
\sigma_{n}^{2} \\
\sigma_{n}^{3} \\
\sigma_{n}^{2 \bigvee 3}
\end{array}\right) .
$$

We drop coordinate 1 but we keep coordinate 4 in our recursion for the reasons explained above. It is easy to check that what was just done is in fact equivalent to the generic way of transforming (4) into (2) which was presented above.

Here it is easy to check that $x_{n}^{(2)} \geq x_{n-1}^{(2)}$ and $x_{n}^{(3)} \geq x_{n-1}^{(3)}$ for all $n \geq 1$, and that this in turn implies that $x_{n}(4) \geq x_{n-1}(4)$. Hence we can take (note that the size of the matrix is $s=3<$ $K L=4)$ :

$$
A_{n}=\left(\begin{array}{ccc}
\sigma_{n}^{2} & \epsilon & \epsilon \\
\epsilon & \sigma_{n}^{3} & \epsilon \\
\sigma_{n}^{2} & \sigma_{n}^{3} & 0
\end{array}\right) \quad B_{n}=\left(\begin{array}{c}
\sigma_{n}^{2} \\
\sigma_{n}^{3} \\
\sigma_{n}^{2 \vee 3}
\end{array}\right)
$$

Similar modifications can be made in FIFO networks where for all $i, x_{n}^{(i)} \geq x_{n-1}^{(i)}$.

Remark 2 Although we will not need this in what follows, we find it useful to stress that one can also associate to all event graphs some token dynamics (see [3] p. 69 and following). If one sees Equation (4) as an extension of Lindley's equation (initially for the G/G/l queue) to event graphs, the token dynamics of event graphs can then be seen as a generalization of that of customers in such a queue.

Property 1 Consider an event graph such that

Al For all $i \in \mathcal{T}$, there exists a tokenless path in the oriented graph $\mathcal{G}$, going from $t_{1}$ to $t_{\text {out }}$ through $i$;

A2 Each transition $i$ is either untimed (with $\sigma_{n}^{i} \equiv 0$ ) or recycled, namely such that there exists a place $p$ with marking 1 such that $p$ is both a predecessor and a successor of $i$ (a natural way of making the event graph FIFO).

Then the network associated with this event graph is monotone-separable.

The proof of separability (the only non immediate property to be proved) is forwarded to Lemma 9 of the appendix.

\subsection{Reducible and Irreducible Event Graphs}

Two transitions of an event graph will be said to belong to the same communication class if there is a directed path in $\mathcal{G}$ from the first to the second and another one from the second to the first. We denote by $\mathcal{C}_{1}, \ldots, \mathcal{C}_{d}$ these communication classes, which form a partition of the set of transitions. 
By construction, these communication classes can be arranged according to a partial order denoted $\prec$. The numbering is assumed to be compatible with this partial order: $\mathcal{C}_{i} \prec \mathcal{C}_{j} \Rightarrow i \leq j$. With this numbering, the matrix in the evolution equation of the event graph has the following block structure:

$$
A_{n}=\left(\begin{array}{ccccccc}
A_{n}(1,1) & \mid & \epsilon & \mid & \epsilon & \mid & \epsilon \\
- & - & - & - & - & - & - \\
A_{n}(2,1) & \mid & A_{n}(2,2) & \mid & \epsilon & \mid & \epsilon \\
- & - & - & - & - & - & - \\
& \vdots & & \vdots & & \vdots & \\
- & - & - & - & - & - & - \\
A_{n}(d, 1) & \mid & A_{n}(d, 2) & \mid & & \mid & A_{n}(d, d)
\end{array}\right),
$$

where each $A_{n}(i, i)$ is an irreducible matrix (corresponding to communication class $\mathcal{C}_{i}$ ).

As the output transition is necessarily in the last communication class ("last" refers here to the partial order $\prec$ ), this choice of numbering can be made compatible with our earlier assumption that the last coordinate is that of the output transition.

\section{Stochastic Assumptions}

\subsection{Model Description and Stochastic Assumptions}

For now on, we consider an event graph as described in $\$ 2.3$, with $m \leq K$ timed transitions, namely $\mathcal{T}_{\text {timed }}=\{t(1), \ldots, t(m)\}$, satisfying the assumptions in Property 1, and with associated recursion:

$$
X_{n}=A_{n} \otimes X_{n-1} \oplus B_{n} \otimes T_{n}
$$

of dimension $s \leq K L$. This means that the matrices $\left\{A_{n}, B_{n}\right\}$ and vectors that are used in the recursion are obtained via two applications $f$ and $g$ such that:

$$
\begin{aligned}
& \begin{array}{cccc}
f: & \mathbb{R}_{+}^{m} & \rightarrow & \mathbb{M}_{(s, s)}\left(\mathbb{R}_{\max }\right) \\
\sigma=\left(\sigma^{1}, \ldots, \sigma^{m}\right) & \mapsto & A(\sigma),
\end{array} \\
& \begin{array}{rllc}
g: & \mathbb{R}_{+}^{m} & \rightarrow & \mathbb{M}_{(s, 1)}\left(\mathbb{R}_{\max }\right) \\
\sigma=\left(\sigma^{1}, \ldots, \sigma^{m}\right) & \mapsto & B(\sigma),
\end{array}
\end{aligned}
$$

via the formula

$$
\begin{aligned}
& f\left(\xi_{n}\right)=A_{n}, \\
& g\left(\xi_{n}\right)=B_{n} .
\end{aligned}
$$

with $\xi_{n}=\left(\sigma_{n}^{t(1)}, \ldots, \sigma_{n}^{t(m)}\right)$.

We now assume that the following independence assumption holds: 
Assumption 1 (IA) The sequences $\left\{\xi_{n}\right\}$ and $\left\{\tau_{n} \equiv T_{n+1}-T_{n}\right\}$ are mutually independent and each of them consists of i.i.d. random variables.

The following assumptions are also assumed to hold

$$
\mathbb{E}\left(\tau_{0}\right) \equiv \lambda^{-1} \equiv a<\infty, \quad \mathbb{E}\left(\sigma_{0}^{t(i)}\right) \equiv b^{t(i)}<\infty \quad \forall i=1, \ldots, m .
$$

This implies in particular $\mathbb{E}\left(Z_{[0,0]}\right)<\infty$.

Under these assumptions, considering the matrices $A_{n}(k, k)$, we have for all $i$ and $j$ :

$$
\frac{\left(A_{-1}(k, k) \otimes A_{-2}(k, k) \otimes \cdots \otimes A_{-n}(k, k)\right)^{(i, j)}}{n} \rightarrow \gamma_{k} \quad \text { both a.s. and in } L_{1}
$$

where $\gamma_{k}$ is a constant referred to as the top Lyapunov exponent of the sequence $\left\{A_{n}(k, k)\right\}$, see theorem 7.27 (p. 325) in [3].

In addition, we assume stability of the system, namely $\max _{k} \gamma_{k}=\gamma<a$ (see [4]).

We will also adopt the following notations:

- if $j \in \mathcal{C}_{i}$, we denote $\gamma_{(j)}=\gamma_{i}$;

- for all transitions $i$, the subset of transitions $j$ such that there is a directed path in $\mathcal{G}$ from $i$ to $j$ is denoted $[\geq i]$

- finally, we define

$$
\Gamma_{(\geq i)}=\max _{k \in[\geq i]} \gamma_{(k)}
$$

The subexponential assumptions are now the following:

Assumption 2 (SE) The service times $\sigma^{t(k)}$ are independent r.v., with respective mean $b^{t(k)}$. There exists a distribution function $F$ on $\mathbb{R}_{+}$such that:

- (SE.1) $F$ is subexponential, with finite first moment $M$.

- (SE.2) The integrated distribution $F^{s}$ of $F$ with the tail

$$
\bar{F}^{s}(x) \equiv 1-F^{s}(x)=\min \left\{1, \int_{x}^{\infty} \bar{F}(u) d u\right\} .
$$

is subexponential.

- (SE.3) The following equivalence holds when $x$ tends to $\infty$ :

$$
\mathbb{P}\left(\sigma_{1}^{t(i)}>x\right) \sim c^{t(i)} \bar{F}(x),
$$

for all $i=1, \ldots, m$ with $\sum_{i=1}^{m} c^{t(i)}=c>0$. 
For $i \notin \mathcal{T}_{\text {timed }}$, we will denote $\forall k, \sigma_{k}^{i}=0$ and $c^{i}=0$. Under (SE.1) and (SE.3), we have (see [7] or [8]):

\section{Lemma 2}

$$
\mathbb{P}\left(\sum_{k=1}^{K} \sigma_{1}^{k}>x\right) \sim \mathbb{P}\left(\max _{1 \leq k \leq K} \sigma_{1}^{k}>x\right) \sim \sum_{k=1}^{K} c^{k} \bar{F}(x)
$$

\subsection{Preliminary Results}

Lemma 3 Under the assumptions of Property 1, there exists some sets $\mathcal{K}_{j}$ such that $\bigcup_{j} \mathcal{K}_{j}=[1 ; s]$ and

$$
B_{n}^{(s)}=\bigoplus_{j} \bigotimes_{k \in \mathcal{K}_{j}} \sigma_{n}^{k}=\max _{j} \sum_{k \in \mathcal{K}_{j}} \sigma_{n}^{k}
$$

Moreover, $\forall j$ there exists only one integer $k(j)$ such that:

$$
\begin{aligned}
\left(A_{n}\right)^{(k(j), k(j))} & \geq \sigma_{n}^{j}, \\
\left(A_{n}\right)^{(s, k(j))} & \geq \sigma_{n}^{j}, \\
\left(B_{n}\right)^{(k(j))} & \geq \sigma_{n}^{j} .
\end{aligned}
$$

The following two properties hold (referred to as (AA') in what follows):

$$
Z_{i}=Z_{[i, i]}=\bigoplus_{j} \bigotimes_{k \in \mathcal{K}_{j}} \sigma_{i}^{k}=\max _{j} \sum_{k \in \mathcal{K}_{j}} \sigma_{i}^{k},
$$

and, when denoting by $Q$ the point process with all its points in 0

$$
Z_{[n, 0]}(Q) \geq \max _{k} \sum_{i=n}^{0} \sigma_{i}^{k} .
$$

Proof :

The first part is proved in Appendix, Section 5.3. Thanks to Lemma 6 in Appendix, we have

$$
Z_{i}=\max _{j} B_{i}^{(j)}=B_{i}^{(s)}
$$

and for the second part:

$$
\begin{aligned}
Z_{[n, 0]}(Q)=\max _{n \leq k \leq 0}\left[\left(A_{0} \otimes \cdots \otimes A_{k+1} \otimes B_{k}\right)^{(s)}\right] & \geq\left(A_{0} \otimes \cdots \otimes A_{n+1} \otimes B_{n}\right)^{(s)} \\
& \geq\left(A_{0}\right)^{(s, k(j))}+\cdots+\left(A_{n+1}\right)^{(k(j), k(j))}+\left(B_{n}\right)^{(k(j))} \\
& \geq \sigma_{0}^{j}+\cdots+\sigma_{n+1}^{j}+\sigma_{n}^{j},
\end{aligned}
$$

for all $j . \triangle$ 
Lemma 4 For all positive integers $L$, let

$$
\hat{s}_{n}=Z_{[L(n-1)+1, L n]}(Q) .
$$

We have

$$
\max _{k} \sum_{i=L(n-1)+1}^{L n} \sigma_{i}^{k} \leq \hat{s}_{n} \leq \sum_{k=1}^{m} \sum_{i=L(n-1)+1}^{L n} \sigma_{i}^{k} .
$$

\section{Proof:}

The first inequality follows from (AA'-2). The second one follows from $Z_{i}=\max _{j} \sum_{k \in \mathcal{K}_{j}} \sigma_{i}^{k} \leq$ $\sum_{k \in[1 ; s]} \sigma_{i}^{k}$, and the sub-additivity of $Z$. $\triangle$

We will assume that assumptions (IA) and (SE) hold throughout this paper without restating it. Moreover $N_{x}$ will denote a non-deceasing integer-valued function tending to infinity such that for all finite real numbers $b$,

$$
\sum_{n=0}^{N_{x}} \bar{F}(x+n b)=o\left(\bar{F}^{s}(x)\right) .
$$

The existence of this function follows from the fact that $F^{s}$ is long-tailed (see [5]).

Property 2 Let $Z$ be the stationary maximal dater of the event graph: $Z \equiv \lim _{n \rightarrow \infty} Z_{[-n, 0]}$. For any $x$ and for $j=1, \ldots, r$, let $\left\{K_{n, x}^{j}\right\}$ be a sequence of events such that

1. for any $n$ and $j$, the event $K_{n, x}^{j}$ and the random variable $\sigma_{-n}^{j}$ are independent;

2. for any $j, \mathbb{P}\left(K_{n, x}^{j}\right) \rightarrow 1$ uniformly in $n \geq N_{x}$ as $x \rightarrow \infty$.

For all sequences $\eta_{n}^{j}, j=1, \ldots, s$, tending to 0 , put

$$
A_{n, x}^{j}=K_{n, x}^{j} \cap\left\{\sigma_{-n}^{j}>x+n\left(a-\gamma+\eta_{n}^{j}\right)\right\}, \quad A_{x}^{j}=\bigcup_{n=N_{x}}^{\infty} A_{n, x}^{j} \quad \text { and } \quad A_{x}=\bigcup_{j=1}^{s} A_{x}^{j} .
$$

Then, as $x \rightarrow \infty$,

$$
\mathbb{P}[Z>x] \sim \mathbb{P}\left[Z>x, A_{x}\right] \sim \sum_{j=1}^{s} \sum_{n \geq N_{x}} \mathbb{P}\left[Z>x, A_{n, x}^{j}\right] .
$$

Proof:

The proof is omitted but uses the same arguments as the proof of Theorem 8 in [5]. The only difference lies in the fact that Condition (AA) in [5] has to be replaced by (AA'), defined in Lemma 3. But under (AA'), (7) of [5] still holds as shown in Lemma 4, which is enough to prove the desired result. $\triangle$ 


\section{Exact Tail Asymptotic}

\section{Theorem 1}

$$
\mathbb{P}(Z>x) \sim\left(\sum_{i=1}^{s} \frac{c^{i}}{a-\Gamma_{(\geq i)}}\right) \bar{F}^{s}(x),
$$

with:

$$
\Gamma_{(\geq i)}=\max _{k \in[\geq i]} \gamma_{(k)}
$$

Proof:

For the sake of simplicity, we give a proof in the case of constant inter-arrival times only. In fact, it was shown in [5] Section 7.3., that the result extends to the stochastic framework we introduced.

Lower bound:

Thanks to Property 2, we have

$$
\mathbb{P}(Z>x) \sim \sum_{n=N_{x}}^{\infty} \sum_{j=1}^{s} \mathbb{P}\left(Z>x, A_{x, n}^{j}\right) .
$$

We have to find appropriate sequences $\left\{K_{n, x}^{j}\right\}$ and $\left\{\eta_{n}^{j}\right\}$.

For all $j$, we have $\left(B_{-n}\right)^{(k(j))} \geq \sigma_{-n}^{j}$. Hence we have

$$
Z \geq \sigma_{-n}^{j}+\left(A_{-1} \otimes A_{-2} \otimes \ldots \otimes A_{-n+1}\right)^{(s, k(j))}-n a .
$$

Consider the events

$$
K_{n, x}^{j}=\left\{\left(A_{-1} \otimes A_{-2} \otimes \ldots \otimes A_{-n+1}\right)^{(s, k(j))} \geq n\left(\Gamma_{(\geq j)}-\eta_{n}^{j}\right)\right\},
$$

and choose a sequence $\eta_{n}^{j} \rightarrow 0$ such that $\mathbb{P}\left[K_{n, x}^{j}\right] \rightarrow 1$ uniformly in $n \geq N_{x}$ as $x \rightarrow \infty$. Then from (9), we have

$$
\begin{aligned}
\mathbb{P}\left(Z>x, A_{x, n}^{j}\right) & \geq \mathbb{P}\left(\sigma_{-n}^{j}>x+n\left(a-\gamma+\eta_{n}^{j}\right), \sigma_{-n}^{j}>x+n a-n\left(\Gamma_{(\geq j)}-\eta_{n}^{j}\right)\right) \\
& \geq(1+o(1)) \mathbb{P}\left(\sigma_{-n}^{j}>x+n\left[a+\eta_{n}^{j}-\min \left(\gamma, \Gamma_{(\geq j)}\right)\right]\right) .
\end{aligned}
$$

But we have for all $j, \Gamma_{(\geq j)} \leq \Gamma_{(\geq 1)}$ and $\gamma=\Gamma_{(\geq 1)}$.

Hence we get an equivalent in $\frac{c^{j}}{a-\Gamma_{(\geq j)}} \bar{F}^{s}(x)$.

Upper bound:

We have

$$
\mathbb{P}\left(Z>x, A_{x}\right)=\sum_{j=1}^{s} \sum_{n \geq N_{x}} \mathbb{P}\left(Z>x, \sigma_{-n}^{j}>x+n\left(a-\gamma+\eta_{n}^{j}\right), K_{x, n}^{j}\right) .
$$


As

$$
\mathbb{P}\left(Z>x, \sigma_{-n}^{j}>x+n\left(a-\gamma+\eta_{n}\right), K_{x, n}^{j}\right) \leq \mathbb{P}\left(\sigma_{-n}^{j}>x+n\left(a-\gamma+\eta_{n}\right)\right),
$$

we have an upper bound in $(1+o(1)) \frac{c^{j}}{a-\Gamma_{(\geq 1)}} \int_{x}^{\infty} \bar{F}(y) d y$.

We consider now the case $\Gamma_{(\geq j)}<\Gamma_{(\geq 1)}$.

We then have the following decomposition:

$$
\begin{aligned}
Z= & \max \left\{Z_{[-n+1 ; 0]} ; \max _{k \geq 0}\left[\left(A_{-1} \otimes \cdots \otimes A_{-n-k+1} \otimes B_{-n-k}\right)^{(s)}-(n+k) a\right]\right\} \\
\equiv & \max \left\{U_{n} ; V_{n}\right\}, \\
V_{n}= & \max \left\{\left(A_{-1} \otimes \cdots \otimes A_{-n+1} \otimes B_{-n}\right)^{(s)}-n a ;\right. \\
& \left.\max _{k \geq 1}\left[\left(A_{-1} \otimes \cdots \otimes A_{-n-k+1} \otimes B_{-n-k}\right)^{(s)}-(n+k) a\right]\right\} \\
\equiv & \max \left\{Z_{n}^{1} ; Z_{n}^{2}\right\} .
\end{aligned}
$$

Thanks to Lemma 10 , we have $Z_{n}^{2} \leq Z_{n}^{1}+R_{n}$, where $R_{n}=Z_{[-\infty,-n-1]}$ is a random variable independent of $\sigma_{-n}^{j}$. Hence we have

$$
\begin{aligned}
V_{n}>x & \Rightarrow Z_{n}^{1}>x \text { or } Z_{n}^{2}>x \\
& \Rightarrow Z_{n}^{1}>x \text { or } Z_{n}^{1}+R_{n}>x \\
& \Rightarrow Z_{n}^{1}+R_{n}>x
\end{aligned}
$$

Hence

$$
\mathbb{P}\left(Z>x, A_{n, x}^{j}\right) \leq \mathbb{P}\left(\max \left\{Z_{n}^{1}+R_{n}, U_{n}\right\}>x, A_{n, x}^{j}\right) .
$$

We will denote $P A_{n}=A_{-1} \otimes \cdots \otimes A_{-n+1}$. We have then

$$
\begin{aligned}
Z_{n}^{1} & =\max \left[Z_{n}^{(\geq j)}, Z_{n}^{(\geq j)^{c}}\right] \quad \text { with } \\
Z_{n}^{(\geq j)} & =\max _{i \in[\geq j]}\left[P A_{n}^{(s, i)}+\left(B_{-n}\right)^{(i)}\right]-n a, \\
Z_{n}^{(\geq j)^{c}} & =\max _{i \in[\geq j]^{c}}\left[P A_{n}^{(s, i)}+\left(B_{-n}\right)^{(i)}\right]-n a .
\end{aligned}
$$

Since $U_{n} \leq Z$ a.s., $\mathbb{P}\left(U_{n} \leq x\right) \rightarrow 1$ uniformly in $n$ as $x \rightarrow \infty$. Since the distribution of $R_{n}=$ $Z_{[-\infty,-n-1]}$ does not depend on $n, R_{n} / n \rightarrow 0$ in probability. Due to the SLLN, $\max _{i \in[\geq j]^{c}}\left[P A_{n}^{(s, i)}+\right.$ $\left.\left(B_{-n}\right)^{(i)}\right] / n \rightarrow c_{j} \leq \gamma$ and $\max _{i \in[\geq j]}\left[P A_{n}^{(s, i)}\right] / n \rightarrow \Gamma_{(\geq j)}$. For $i \in[\geq j]$, we have $\left(B_{-n}\right)^{(i)} \leq$ $\sigma_{-n}^{j}+\sum_{k \neq j} \sigma_{-n}^{k}$. We denote $\zeta_{n}^{j}=\sum_{k \neq j} \sigma_{-n}^{k}$, we have $\zeta_{n}^{j} / n \rightarrow 0$ in probability. Therefore, there exists a sequence $\epsilon_{n} \downarrow 0, n \epsilon_{n} \rightarrow \infty$ such that

$$
\begin{array}{r}
\mathbb{P}\left\{U_{n} \leq x, R_{n} \leq n \epsilon_{n}, \max _{i \in[\geq j]^{c}}\left[P A_{n}^{(s, i)}+\left(B_{-n}\right)^{(i)}\right] \leq n\left(\gamma+\epsilon_{n}\right),\right. \\
\left.\max _{i \in[\geq j]}\left[P A_{n}^{(s, i)}\right] \leq n\left(\Gamma_{(\geq j)}+\epsilon_{n}\right), \zeta_{n}^{j} \leq n \epsilon_{n}\right\} \rightarrow 1
\end{array}
$$

$\operatorname{RR} n^{\circ} 4952$ 
uniformly in $n \geq N_{x}$ as $x \rightarrow \infty$. Denote the latter event $K_{n, x}^{j}$. For $i \in[\geq j]^{c}$, the random variables $\left(B_{-n}\right)^{(i)}$ and $\sigma_{-n}^{j}$ are independent, hence $K_{n, x}^{j}$ is independent of $\sigma_{-n}^{j}$. Moreover, observe that on $K_{n, x}^{j}$, we have

$$
\begin{aligned}
\left\{\max \left\{Z_{n}^{1}+R_{n}, U_{n}\right\}>x\right\} & =\left\{Z_{n}^{1}+R_{n}>x\right\} \\
& \subset\left\{n\left(\gamma+\epsilon_{n}\right)-n a+R_{n}>x\right\} \cup\left\{n\left(\Gamma_{(\geq j)}+\epsilon_{n}\right)+n \epsilon_{n}+\sigma_{-n}^{j}+n \epsilon_{n}-n a>x\right\} .
\end{aligned}
$$

Put $\eta_{n}^{j}=-3 \epsilon_{n}$. Then

$$
\begin{aligned}
\mathbb{P}\left(Z>x, A_{n, x}^{j}\right) \leq & \mathbb{P}\left(R_{n}>x+n\left(a-\gamma-\epsilon_{n}, K_{n, x}^{j}\right) \mathbb{P}\left(\sigma_{-n}^{j}>x+n\left(a-\gamma+\eta_{n}^{j}\right)\right)\right. \\
& \quad+\mathbb{P}\left(\sigma_{-n}^{j}>x+n\left(a-\Gamma_{(\geq j)}-3 \epsilon_{n}\right), K_{n, x}^{j}\right) \\
= & o(1) \mathbb{P}\left(\sigma_{-n}^{j}>x+n\left(a-\gamma+\eta_{n}^{j}\right)\right)+(1+o(1)) \mathbb{P}\left(\sigma_{-n}^{j}>x+n\left(a-\Gamma_{(\geq j)}+\eta_{n}^{j}\right)\right),
\end{aligned}
$$

and the desired asymptotics follows. $\triangle$

\section{Appendix}

\subsection{Preliminary Properties}

We start with some preliminary technical lemmas which receive a natural interpretation in terms of paths of maximal weight in oriented weighted graphs. We use the notation $|\mathcal{T}|=K, L$ is the maximal value of the initial marking, and $s \leq K L$ is the size of the matrix in recursion (2) after simplification (see Remark 1).

\section{Lemma 5 Properties of $a_{0}^{*}$ :}

1. $\forall i, j, k \quad\left(a_{0}^{*}\right)^{(i, j)}+\left(a_{0}^{*}\right)^{(j, k)} \leq\left(a_{0}^{*}\right)^{(i, k)}$;

2. $\forall i, \quad\left(a_{0}^{*}\right)^{(i, 1)} \geq \max _{j}\left(a_{0}\right)^{(i, j)}$;

3. $\forall i, \quad\left(a_{0}^{*}\right)^{(K, i)} \geq 0$.

\section{Proof:}

We denote by $\mathcal{G}_{0}$ the weighted oriented graph associated to $\left(a_{0}\right)$ (see $\S 2.3$ of [3]). The element $\left(a_{0}^{*}\right)^{(i, j)}$ is equal to the maximum of the weights of all tokenless paths of the graph going from $j$ to $i$.

The triangular structure of $\left(a_{0}\right)$ means there is no tokenless path from $i$ to $j$ if $i \geq j$. Moreover assumption A1 in property 1 ensures that for all $i$, there exists a tokenless path of $\mathcal{G}_{0}$ going from 1 to $K$ through $i$. So we have $\left(a_{0}^{*}\right)^{(i, 1)} \geq 0$ and $\left(a_{0}^{*}\right)^{(K, i)} \geq 0$, which proves 3 . Moreover, as $\left(a_{0}^{*}\right)^{(i, 1)}$ is the maximum of the weights of all paths going from 1 to $i$, we have

$$
\left(a_{0}^{*}\right)^{(i, 1)}=\max _{j}\left(a_{0}\right)^{(i, j)}+\left(a_{0}^{*}\right)^{(j, 1)} \geq \max _{j}\left(a_{0}\right)^{(i, j)},
$$


which proves 2. Finally, as the maximal weight of the tokenless paths from $i$ to $k$ is larger than that of the paths from $i$ to $j$ plus that of the paths from $j$ to $k$, we get 1 .

$\triangle$

Lemma 6 For all $k \leq n$ and for all $j,\left(D_{[k, n]} \otimes B_{k}\right)^{(j)} \leq\left(D_{[k, n]} \otimes B_{k}\right)^{(s)}$.

Proof:

We will prove this lemma on the matrices $A_{n}$ and $B_{n}$ before simplification, which is enough to prove the result. We denote by $s=K L$ the size of the matrices.

- Step 1 For all $j, n, B_{n}^{(j)} \leq B_{n}^{(s)}$.

In what follows, we omit the dependence in $n$ when not necessary. In (4), the variable $T_{n}$ only arises in $x_{n}(1)=\cdots \oplus T_{n}$. Since $b=(0, \epsilon, \cdots, \epsilon)^{\prime}$, we have $\bar{b}(n)=\left(a_{0}(n)^{*}\right)^{(., 1)}$. Hence we only have to show that $\left(a_{0}^{*}\right)^{(j, 1)} \leq\left(a_{0}^{*}\right)^{(K, 1)}$. But we know from Lemma 5 that

$$
\left(a_{0}^{*}\right)^{(K, 1)} \geq\left(a_{0}^{*}\right)^{(K, j)}+\left(a_{0}^{*}\right)^{(j, 1)} \text { and }\left(a_{0}^{*}\right)^{(K, j)} \geq 0 .
$$

- Step 2 For all $i, j, l, n, \bar{a}_{l}(n)^{(j, i)} \leq \bar{a}_{l}(n)^{(K, i)}$.

In what follows, we omit the indices $l$ and $n$. We have $\bar{a}^{(j, i)}=\max _{k}\left[\left(a_{0}^{*}\right)^{(j, k)}+a^{(k, i)}\right]$ but for all $k$ and $i$ :

$$
\begin{aligned}
\left(a_{0}^{*}\right)^{(K, j)}+\left(a_{0}^{*}\right)^{(j, k)} & \leq\left(a_{0}^{*}\right)^{(K, k)} \\
\left(a_{0}^{*}\right)^{(K, j)}+\left(a_{0}^{*}\right)^{(j, k)}+a^{(k, i)} & \leq\left(a_{0}^{*}\right)^{(K, k)}+a^{(k, i)},
\end{aligned}
$$

which implies $\bar{a}^{(j, i)} \leq \bar{a}^{(K, i)}$.

- Step 3 For all $j, k, n,\left(A_{n} \otimes \cdots \otimes A_{k+1} \otimes B_{k}\right)^{(j)} \leq\left(A_{n} \otimes \cdots \otimes A_{k+1} \otimes B_{k}\right)^{(s)}$.

Given a vector $z=\left(z_{1}, z_{2}, \ldots, z_{L}\right)=\left(z^{(1)}, \ldots, z^{(s)}\right)$, where each $z_{i}$ is of dimension $K$, we have:

$$
\begin{aligned}
\left(A_{n} \otimes z\right)^{(j)} & =\max _{l}\left[\left(A_{n}\right)^{(j, l)}+z^{(l)}\right] \\
& = \begin{cases}z^{(j+K)} & \text { for } j \in[1,(L-1) K], \\
\bigoplus_{i=1}^{L}\left(\bar{a}_{i}\right)^{(j, .)} \otimes z_{L+1-i} & \text { for } j \in[(L-1) K+1, L K] .\end{cases}
\end{aligned}
$$

But we have shown that

$$
\begin{aligned}
\left(\bar{a}_{i}\right)^{(j, .)} \otimes z_{L+1-i} & =\max _{l}\left[\left(\bar{a}_{i}\right)^{(j, l)}+z_{L+1-i}^{(l)}\right] \\
& \leq \max _{l}\left[\left(\bar{a}_{i}\right)^{(K, l)}+z_{L+1-i}^{(l)}\right] \\
& =\left(\bar{a}_{i}\right)^{(K, .)} \otimes z_{L+1-i} .
\end{aligned}
$$

Hence for $j \in[(L-1) K+1, L K]$, we have $\left(A_{n} \otimes z\right)^{(j)} \leq\left(A_{n} \otimes z\right)^{(s)}$ for all $z$. 
We now show the property by induction on $n$. For $n=k$, the result is true : $B_{k}^{(j)} \leq B_{k}^{(s)}$. We suppose the result true for $n$.

Let $z=D_{[k, n]} \otimes B_{k}$. Thanks to the induction hypothesis, we have $z^{(j)} \leq z^{(s)}, \forall j$. W.l.o.g, we can assume that $\left(A_{n+1}\right)^{(s, s)} \geq 0$. Then, we have

$$
\left(A_{n+1}\right)^{(s, .)} \otimes z=\max _{i}\left[\left(A_{n+1}\right)^{(s, i)}+z^{(i)}\right] \geq z^{(s)} .
$$

Hence

$$
\left(A_{n+1} \otimes z\right)^{(s)} \geq z^{(s)} \geq\left(A_{n+1} \otimes z\right)^{(i)} \quad \text { for } \quad i \in[1,(L-1) K]
$$

since $\left(A_{n+1} \otimes z\right)^{(i)}=z^{(i+K)} \leq z^{(s)}$, by the induction hypothesis. But $A_{n+1} \otimes z=D_{[k, n+1]} \otimes$ $B_{k}$, and the result follows.

Lemma 7 For all $n$ and $i$, we have

$$
\max _{j}\left(A_{n}\right)^{(i, j)} \leq\left[\left(B_{n}\right)^{(i)}\right]^{+} .
$$

Proof:

In view of the definition of $A_{n}$ and $B_{n}$ (see (6)), it is enough to prove that for all $k \geq 1$ and all $i$,

$$
\max _{j}\left(\bar{a}_{k}\right)^{(i, j)} \leq(\bar{b})^{(i)} \text {. }
$$

We first show that for all $k \geq 1$ :

$$
\max _{j}\left(a_{0}\right)^{(i, j)} \geq \max _{j}\left(a_{k}\right)^{(i, j)} \quad \text { for } \quad i \neq 1 \text {. }
$$

For $i \neq 1$, thanks to $\mathrm{A} 1$, there exists a transition $j \in \mathcal{T}, j \neq i$ such that there is a two hop tokenless path from $j$ to $i$. So we have $\max _{j}\left(a_{0}\right)^{(i, j)}=\sigma^{i} \geq 0$. Moreover, we have

$$
\forall k, \quad \max _{j}\left(a_{k}\right)^{(i, j)}= \begin{cases}\sigma^{i} & \text { if } \exists p \text { predecessor of } i \text { with marking } k ; \\ \epsilon & \text { else. }\end{cases}
$$

Hence, we get (12).

Let $v$ and $\bar{v}$ be the vectors defined by $v^{(i)}=\max _{j}\left(a_{k}\right)^{(i, j)}$ and $\bar{v}^{(i)}=\max _{j}\left(\overline{a_{k}}\right)^{(i, j)}$. We have

$$
\bar{v}=a_{0}^{*} \otimes a_{k} \otimes \mathbf{0}=a_{0}^{*} \otimes v .
$$

We have to prove (11), that is $\bar{v} \leq \bar{b}$. We have $v^{(1)} \leq 0$, so that $\bar{v}^{(1)}=v^{(1)} \leq \bar{b}^{(1)}=0$. We now show that $\bar{v}^{(i)} \leq \bar{b}^{(i)}$ for $i>1$. In view of (12) and of Property 2 of Lemma 5 , for $i \neq 1$ :

$$
v^{(i)} \leq\left(a_{0}^{*}\right)^{(i, 1)}=\bar{b}^{(i)} .
$$


We have then

$$
\begin{aligned}
v^{(i)}+\left(a_{0}^{*}\right)^{(j, i)} & \leq\left(a_{0}^{*}\right)^{(j, i)}+\left(a_{0}^{*}\right)^{(i, 1)} \\
& \leq\left(a_{0}^{*}\right)^{(j, 1)} \\
& =\bar{b}^{(j)}
\end{aligned}
$$

But we have

$$
\bar{v}^{(i)}=\max \left[v^{(i)}, v^{(i-1)}+\left(a_{0}^{*}\right)^{(i, i-1)}, v^{(i-2)}+\left(a_{0}^{*}\right)^{(i, i-2)}, \ldots\right] .
$$

Finally, we get

$$
\bar{v}^{(i)} \leq \bar{b}^{(i)}
$$

$\triangle$

Remark 3 Concerning the simplification of the matrix $A_{n}$.

We consider the oriented graph $\mathcal{G}_{0}$ associated to $a_{0}$. For $i \in \mathcal{T}$, we denote by prem $(i)$ the set of timed transitions $j$ such that there exists a path from $j$ to $i$ in $\mathcal{G}_{0}$ and such that any predecessor of $j$ in $\mathcal{G}_{0}$ is not timed. In particular, if $i$ is not timed and if no transition in $\pi_{0}^{+}(i)$ (set of ascendants, see [3] p.36) is timed, then prem $(i)=\emptyset$. We denote then $\mathcal{T}_{\text {temp }}=\bigcup_{i} \sigma_{0}^{*}(\operatorname{prem}(i))$.

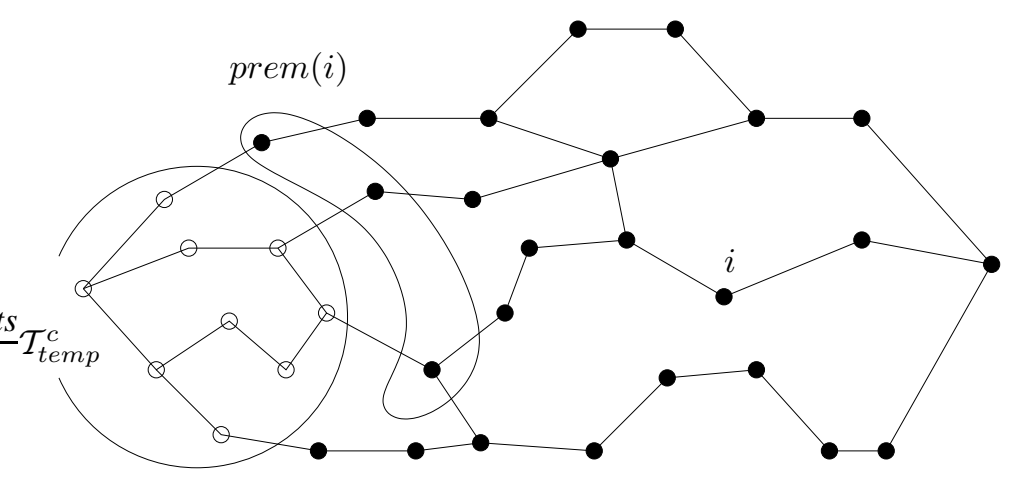

We then have the following property: the columns of the matrices $\bar{a}_{k}$ numbered $i \in\left(\mathcal{T}_{\text {temp }}\right)^{c}$ are null.

Indeed, if $i \in\left(\mathcal{T}_{\text {temp }}\right)^{c}$, we have $x_{n}^{(i)}=T_{n}$ for all $n$. First suppose there exists a subscript $j$ such that $\left(a_{k}\right)^{(j, i)} \neq \epsilon$ for a certain $k \geq 1$. We then have in recursion $(4),\left(x_{n}\right)^{(j)}=\left[\left(x_{n-k}\right)^{(i)}+\sigma_{n}^{j}\right] \oplus \ldots$ But we know that there exists a tokenless path $\left(j_{l}=j, \ldots, j_{1}=1\right)$ going from 1 to $j$. We then have $\left(x_{n}\right)^{(j)} \geq T_{n}+\sigma_{n}^{j_{1}}+\cdots+\sigma_{n}^{j_{l}}$. We see that

$$
T_{n}+\sigma_{n}^{j_{1}}+\cdots+\sigma_{n}^{j_{l}} \geq\left(x_{n-k}\right)^{(i)}+\sigma_{n}^{j}=T_{n-k}+\sigma_{n}^{j} .
$$

Hence, we can assume that for $i \in\left(\mathcal{T}_{\text {temp }}\right)^{c}, \forall j, k \quad\left(a_{k}\right)^{(j, i)}=\epsilon$. This implies $\left(\bar{a}_{k}\right)^{(j, i)}=$ $\max _{l}\left[\left(a_{0}^{*}\right)^{(j, l)}+\left(a_{k}\right)^{(l, i)}\right]=\epsilon$. 
This implies that the columns of matrix $A_{n}$ that are numbered $i \in\left(\mathcal{T}_{\text {temp }}\right)^{c}$ modulo $K$ are dropped. We take the same notation as in the last proof $\bar{v}_{k}^{(i)}=\max _{j}\left(\bar{a}_{k}\right)^{(i, j)}$.

We show that for all $i \in \mathcal{T}_{\text {temp }}$

$$
\bar{v}_{1}^{(i)}=\bar{b}^{(i)}
$$

If $i$ is a timed transition, we have

$$
\max _{j}\left(a_{1}\right)^{(i, j)}=\sigma^{i}
$$

Indeed, thanks to the FIFO assumption, we have $:\left(a_{1}\right)^{(i, i)}=\sigma^{i} \geq 0$.

We then have for $i \in \mathcal{T}_{\text {temp }}$ :

$$
\begin{aligned}
{\overline{v_{1}}}^{(i)} & =\max _{l}\left[\left(a_{0}^{*}\right)^{(i, l)}+\left(v^{1}\right)^{(l)}\right] \\
& \geq \max _{j \in \operatorname{prem}(i)}\left[\left(a_{0}^{*}\right)^{(i, j)}+\left(v^{1}\right)^{(j)}\right] \\
& =\max _{j \in \operatorname{prem}(i)}\left[\sigma^{j}+\left(a_{0}^{*}\right)^{(i, j)}\right] .
\end{aligned}
$$

But we have

$$
\begin{aligned}
\bar{b}_{i} & =\left(a_{0}^{*}\right)^{(i, 1)} \\
& =\max _{j \in \operatorname{prem}(i)}\left[\left(a_{0}^{*}\right)^{(j, 1)}+\left(a_{0}^{*}\right)^{(i, j)}\right] \\
& =\max _{j \in \operatorname{prem}(i)}\left[\sigma^{j}+\left(a_{0}^{*}\right)^{(i, j)}\right] .
\end{aligned}
$$

We then have ${\overline{v_{1}}}^{(i)}=\bar{b}^{(i)}$, for $i \in \mathcal{T}_{\text {temp }}$.

We have $B_{l+1}^{(i)}=\epsilon \Rightarrow \max _{j} A_{l}^{(i, j)} \geq 0$. Hence the last lemma implies the following result: when we drop some coordinates while simplifying the matrix $A_{n}$, we get that there exist two subsets of coordinates $I$ and $J$ such that:

$$
\begin{gathered}
\forall i \in I \subset[(L-1) K+1, L K], \quad \max _{j}\left(A_{l}\right)^{(i, j)} \leq\left(B_{l}\right)^{(i)} \\
\forall i \in J \subset[1,(L-1) K], \quad\left(B_{l}\right)^{(i)}=\epsilon \quad \max _{j}\left(A_{l}\right)^{(i, j)}=0 .
\end{gathered}
$$

Lemma 8 For any $m<n$, we have

$$
\max _{j \in J}\left(D_{[m, n]}\right)^{(s, j)} \leq \max _{j \in I}\left(D_{[m, n]}\right)^{(s, j)} .
$$


Proof:

We have

$$
\left(D_{[m, n+1]}\right)^{(i, j)}=\max _{l}\left[\left(A_{n+1}\right)^{(i, l)}+\left(D_{[m, n]}\right)^{(l, j)}\right] .
$$

We will prove by induction that

$$
\forall l, \quad \max _{j \in J}\left(D_{[m, n]}\right)^{(l, j)} \leq \max _{j \in I}\left(D_{[m, n]}\right)^{(l, j)} .
$$

For $n=m+1$, we only have to prove that for all $i \in \mathcal{T}_{\text {temp }}$ :

$$
\max _{j}\left(\bar{a}_{1}\right)^{(i, j)} \geq \max _{j}\left(\bar{a}_{k}\right)^{(i, j)} \quad \forall k .
$$

With our notations, this means

$$
{\overline{v_{1}}}^{(i)} \geq{\overline{v_{k}}}^{(i)}
$$

But we saw that $\bar{v}^{(i)} \leq \bar{b}^{(i)}$ and thanks to last remark, we have ${\overline{v_{1}}}^{(i)}=\bar{b}^{(i)}$ for $i \in \mathcal{T}_{\text {temp }}$. Assume now that

$$
\max _{j \in J}\left(D_{[m, n]}\right)^{(l, j)} \leq \max _{j \in I}\left(D_{[m, n]}\right)^{(l, j)} .
$$

We have then

$$
\begin{aligned}
\max _{j \in J}\left(D_{[m, n+1]}\right)^{(l, j)} & =\max _{j \in J} \max _{i}\left[\left(A_{n+1}\right)^{(l, i)}+\left(D_{[m, n]}\right)^{(i, j)}\right] \\
& \leq \max _{j \in I} \max _{i}\left[\left(A_{n+1}\right)^{(l, i)}+\left(D_{[m, n]}\right)^{(i, j)}\right] \\
& =\max _{j \in I}\left(D_{[m, n]}\right)^{(l, j)} .
\end{aligned}
$$

\subsection{Separability}

Lemma 9 Separability: Assume that the following conditions hold:

1. $\forall k \leq n, \quad \forall j, \quad\left(D_{[k, n]} \otimes B_{k}\right)^{(j)} \leq\left(D_{[k, n]} \otimes B_{k}\right)^{(s)}$.

2. $\forall l, \quad \max _{j} A_{l}^{(i, j)} \leq\left[B_{l}^{(i)}\right]^{+} \quad \forall i$.

3. $\forall m \leq n, \quad \max _{j \in J} D_{[m, n]}^{(s, j)} \leq \max _{i \in I} D_{[m, n]}^{(s, i)}$

We have then for $m \leq l<n$ such that $X\left(N_{[m, l]}\right) \leq T_{l+1}$ :

$$
X\left(N_{[m, n]}\right)=X\left(N_{[l+1, n]}\right) .
$$

$\mathrm{RR} \mathrm{n}^{\circ} 4952$ 


\section{Proof:}

From 1, we have

$X\left(N_{[m, n]}\right)=\bigoplus_{m \leq k \leq l}\left(A_{n} \otimes \cdots \otimes A_{k+1} \otimes B_{k} \otimes T_{k}\right)^{(s)} \oplus \bigoplus_{l+1 \leq k \leq n}\left(A_{n} \otimes \cdots \otimes A_{k+1} \otimes B_{k} \otimes T_{k}\right)^{(s)}$.

So it is enough to show that

$\left(A_{n} \otimes \cdots \otimes A_{l+2} \otimes A_{l+1} \otimes A_{l} \otimes \cdots \otimes A_{k+1} \otimes B_{k} \otimes T_{k}\right)^{(s)} \leq\left(A_{n} \otimes \cdots \otimes A_{l+2} \otimes B_{l+1} \otimes T_{l+1}\right)^{(s)}$ or equivalently

$$
\left(D_{[l+1, n]} \otimes A_{l+1} \otimes D_{[k, l]} \otimes B_{k} \otimes T_{k}\right)^{(s)} \leq\left(D_{[l+1, n]} \otimes B_{l+1} \otimes T_{l+1}\right)^{(s)} .
$$

For simplicity of notation, we will denote:

$$
\begin{aligned}
D_{1} & =D_{[l+1, n]} \\
A & =A_{l+1} \\
D_{2} & =D_{[k, l]} \\
U & =B_{k} \\
V & =B_{l+1} .
\end{aligned}
$$

With this notation, we have to show that

$$
\left(D_{1} \otimes A \otimes D_{2} \otimes U \otimes T_{k}\right)^{(s)} \leq\left(D_{1} \otimes V \otimes T_{l+1}\right)^{(s)} .
$$

Moreover, we have

$$
\begin{aligned}
X\left(N_{[m, l]}\right) \leq T_{l+1} & \Leftrightarrow \quad \forall k \geq m \quad A_{l} \otimes \cdots \otimes A_{k+1} \otimes B_{k} \otimes T_{k} \leq T_{l+1} \\
& \Leftrightarrow \quad \forall k \geq m \quad D_{2} \otimes U \otimes T_{k} \leq T_{l+1} \\
& \Leftrightarrow \quad \forall k \geq m \quad \max _{i, j}\left[D_{2}^{(i, j)}+U^{(j)}+T_{k}\right] \leq T_{l+1} .
\end{aligned}
$$

Under this condition, we have

$$
\begin{aligned}
\left(D_{1} \otimes A \otimes D_{2} \otimes U \otimes T_{k}\right)^{(s)} & =\max _{x, y, z}\left[D_{1}^{(s, x)}+A^{(x, y)}+D_{2}^{(y, z)}+U^{(z)}+T_{k}\right] \\
& \leq \max _{x, y}\left[D_{1}^{(s, x)}+A^{(x, y)}\right]+\max _{y, z}\left[D_{2}^{(y, z)}+U^{(z)}+T_{k}\right] \\
& \leq \max _{x, y}\left[D_{1}^{(s, x)}+A^{(x, y)}+T_{l+1}\right] .
\end{aligned}
$$

So it is enough to prove that $\max _{x, y}\left[D_{1}^{(K, x)}+A^{(x, y)}\right] \leq \max _{x}\left[D_{1}^{(K, x)}+V^{(x)}\right]$. But we have

$$
\begin{aligned}
\max _{x, y}\left[D_{1}^{(s, x)}+A^{(x, y)}\right] & =\max \left\{\max _{x \in I}\left[D_{1}^{(s, x)}+\max _{y} A^{(x, y)}\right], \max _{x \in J}\left[D_{1}^{(s, x)}+\max _{y} A^{(x, y)}\right]\right\} \\
& \leq \max \left\{\max _{x \in I}\left[D_{1}^{(s, x)}+V^{(x)}\right], \max _{x \in J}\left[D_{1}^{(s, x)}\right]\right\} \\
& \leq \max _{x \in I}\left[D_{1}^{(s, x)}+V^{(x)}\right]=\max _{x}\left[D_{1}^{(s, x)}+V^{(x)}\right],
\end{aligned}
$$


where we used $2, \max _{j}\left[A^{(i, j)}\right] \leq\left(V^{(i)}\right)^{+}$, to get the first inequality and 3 , which implies $\max _{x \in J}\left[D_{1}^{(s, x)}\right] \leq$ $\max _{x \in I}\left[D_{1}^{(s, x)}\right]$, to get the second one.

$\triangle$

\subsection{Two Technical Lemmas}

\section{Proof of Lemma 3:}

The first property is a mere rewriting of the definition of $\bar{b}_{n}=a_{0}(n)^{*} \otimes b$. Remark 3, which gives the relation between the matrices $\bar{a}_{1}$ and $a_{0}^{*}$, allows one to establish the last properties. Indeed, the maximum in $\left(v_{1}\right)^{(i)}=\max _{j}\left(a_{1}\right)^{(i, j)}$ is on the diagonal. Moreover, we have $\left(\bar{a}_{1}\right)^{(k, k)}=$ $\max _{i}\left[\left(a_{0}^{*}\right)^{(k, i)}+\left(a_{1}\right)^{(i, k)}\right] \geq\left(a_{1}\right)^{(k, k)}$, which ensures the existence of $k(j)$ such that $\left(B_{n}\right)^{(k(j))} \geq$ $\sigma_{n}^{j}$ and $\left(A_{n}\right)^{(k(j), k(j))} \geq \sigma_{n}^{j}$ because the diagonal terms of $\bar{a}_{1}$ are diagonal terms of $A$ too.

Moreover, we have

$$
\begin{aligned}
\left(\bar{a}_{1}\right)^{(s, k)} & =\max _{i}\left[\left(a_{0}^{*}\right)^{(s, i)}+\left(a_{1}\right)^{(i, k)}\right] \\
& \geq\left(a_{0}^{*}\right)^{(s, k)}+\left(a_{1}\right)^{(k, k)} \\
& \geq\left(a_{1}\right)^{(k, k)}
\end{aligned}
$$

and then, we have $\left(A_{n}\right)^{(s, k(j))} \geq \sigma_{n}^{j}$.

$\triangle$

We will denote for $a>0$ and $u \leq v$ :

$$
Z_{[u, v]}=\max _{u \leq i \leq v}\left[\left(D_{[i, v]} \otimes B_{i}\right)^{(s)}-(v-i) a\right] .
$$

Lemma 10 We denote for $n \geq 1$ :

$$
\begin{aligned}
& Z_{n}^{1}=\left(A_{0} \otimes \cdots \otimes A_{-n+1} \otimes B_{-n}\right)^{(s)}-n a, \\
& Z_{n}^{2}=\max _{k \geq 0}\left[\left(A_{0} \otimes \cdots \otimes A_{-n-k+1} \otimes B_{-n-k}\right)^{(s)}-(n+k) a\right] .
\end{aligned}
$$

We have then

$$
Z_{n}^{2} \leq Z_{n}^{1}+Z_{[-\infty,-n-1]}
$$

Proof:

We have only to prove that

$$
\tilde{Z}_{n}^{2} \leq Z_{n}^{1}+Z_{[-\infty,-n-1]},
$$

with $\tilde{Z}_{n}^{2}=\max _{k \geq 1}\left[\left(A_{-1} \otimes \cdots \otimes A_{-n-k+1} \otimes B_{-n-k}\right)^{(s)}-(n+k) a\right]$. We will assume that $k \geq 1$ in what follows and we denote:

$$
\begin{aligned}
D_{1} & =A_{0} \otimes \cdots \otimes A_{-n+1}, \\
D B_{k} & =D_{[-n-k,-n-1]} \otimes B_{-n-k}, \\
Z^{k, 2} & =\left(D_{1} \otimes A_{-n} \otimes D B_{k}\right)^{(s)}-(n+k) a .
\end{aligned}
$$

$\operatorname{RR} \mathrm{n}^{\circ} 4952$ 
With this notation, we have $Z_{n}^{1}=\left(D_{1} \otimes B_{-n}\right)^{(s)}-n a$ and $\tilde{Z}_{n}^{2}=\max _{k \geq 1}\left[Z^{k, 2}\right]$. We have then

$$
\begin{aligned}
Z^{k, 2} & =\max _{i, j}\left[D_{1}^{(s, i)}+\left(A_{-n}\right)^{(i, j)}+D B_{k}^{(j)}\right]-(n+k) a \\
& \leq \max _{i, j}\left[D_{1}^{(s, i)}+\left(A_{-n}\right)^{(i, j)}\right]+\max _{j}\left[D B_{k}^{(j)}\right]-(n+k) a .
\end{aligned}
$$

First show that

$$
\max _{i}\left[D_{1}^{(s, i)}+\left(A_{-n}\right)^{(i, j)}\right] \leq \max _{i}\left[D_{1}^{(s, i)}+\left(B_{-n}\right)^{(i)}\right]
$$

Indeed, thanks to condition 2, we have $\max _{i \in I}\left[D_{1}^{(s, i)}+\left(A_{-n}\right)^{(i, j)}\right] \leq \max _{i \in I}\left[D_{1}^{(s, i)}+\left(B_{-n}\right)^{(i)}\right]$. We have then only to prove that $\max _{i \in J}\left[D_{1}^{(s, i)}+\left(A_{-n}\right)^{(i, j)}\right] \leq \max _{i \in I}\left[D_{1}^{(s, i)}+\left(B_{-n}\right)^{(i)}\right]$.

But we have $\max _{i \in J}\left[D_{1}^{(s, i)}+\left(A_{-n}\right)^{(i, j)}\right]=\max _{i \in J}\left[D_{1}^{(s, i)}\right]$ because $\left(A_{-n}\right)^{(i, j)}=0$ for $i \in J$. Moreover we have $\max _{i \in J}\left[D_{1}^{(s, i)}\right] \leq \max _{i \in I}\left[D_{1}^{(s, i)}\right] \leq \max _{i \in I}\left[D_{1}^{(s, i)}+\left(B_{-n}\right)^{(i)}\right]$ and the equality follows.

Finally, we have

$$
Z^{k, 2} \leq Z_{n}^{1}+\max _{j}\left[D B_{k}^{(j)}\right]-k a
$$

But $\left[D B_{k}^{(j)}\right]=\left(D_{[-n-k,-n-1]} \otimes B_{-n-k}\right)^{(j)} \leq\left(D_{[-n-k,-n-1]} \otimes B_{-n-k}\right)^{(s)}$, and we have then $Z^{k, 2} \leq Z_{n}^{1}+\left(D_{[-n-k,-n-1]} \otimes B_{-n-k}\right)^{(s)}-k a$, and

$$
\begin{aligned}
Z_{n}^{2} & \leq Z_{n}^{1}+\max _{k \geq 1}\left[\left(D_{[-n-k,-n-1]} \otimes B_{-n-k}\right)^{(s)}-k a\right] \\
& \leq Z_{n}^{1}+Z_{[-\infty,-n-1]} .
\end{aligned}
$$

\section{References}

[1] S. Asmussen, H. Schmidli, V. Schmidt, Tail probabilities for non-standard risk and queueing processes with subexponential jumps, Adv. Appl. Prob. 31 (1999) 422-447.

[2] H. Ayhan, Z. Palmowski and S. Schlegel (2002) Cyclic Queueing Networks with Subexponential Service Times private communication.

[3] F. Baccelli, G. Cohen, G.J. Olsder and J.P. Quadrat (1992) Synchronization and Linearity, Wiley.

[4] F. Baccelli and S. Foss (1995) On the Saturation Rule for the Stability of Queues. J. Appl. Prob., 32, pp. 494-507. 
[5] F. Baccelli and S. Foss (2003) Moments and tails in monotone-separable stochastic networks. Rapport INRIA 4197, Annals of Applied Probability (to appear).

[6] F. Baccelli, S. Foss and M. Lelarge, Tails in Generalized Jackson Networks with Subexponential Service Distributions. In preparation.

[7] F. Baccelli, S. Schlegel, and V. Schmidt (1999), Asymptotics of Stochastic Networks with Sub-exponential Service Times. QUESTA, 33, pp. 205-232.

[8] C.M. Goldie, C. Klüppelberg, Subexponential distributions, in A Practical Guide to Heavy Tails : Statistical Techniques and Applications, eds R.J. Adler, R.E. Feldman. M.S. Taqqu, Birkhäuser, 1997, pp. 435-459.

[9] C. Klüppelberg, Subexponential distributions and integrated tails, J. Appl. Prob. 25 (1988) 132-141.

[10] A.G Pakes, On the tails of waiting-time distributions, J. Appl. Prob. 12 (1975) 555-564.

[11] N. Veraverbeke (1977), Asymptotic behavior of Wiener-Hopf factors of a random walk. Stoch. Proc. Appl., 5, pp. 27-37. 
Unité de recherche INRIA Rocquencourt Domaine de Voluceau - Rocquencourt - BP 105 - 78153 Le Chesnay Cedex (France)

Unité de recherche INRIA Lorraine : LORIA, Technopôle de Nancy-Brabois - Campus scientifi que 615, rue du Jardin Botanique - BP 101 - 54602 Villers-lès-Nancy Cedex (France)

Unité de recherche INRIA Rennes : IRISA, Campus universitaire de Beaulieu - 35042 Rennes Cedex (France)

Unité de recherche INRIA Rhône-Alpes : 655, avenue de l'Europe - 38330 Montbonnot-St-Martin (France)

Unité de recherche INRIA Sophia Antipolis : 2004, route des Lucioles - BP 93 - 06902 Sophia Antipolis Cedex (France) 